\title{
MicroRNA-138-5p controls sensitivity of nasopharyngeal carcinoma to radiation by targeting EIF4EBP1
}

\author{
WEI GAO $^{1 *}$, JACKY WEI KEI LAM ${ }^{1 *}$, JOHN ZENG-HONG LI ${ }^{1,2}$, SI-QI CHEN $^{1}$, \\ RAYMOND KING-YIN TSANG ${ }^{1}$, JIMMY YU-WAI CHAN ${ }^{1}$ and THIAN-SZE WONG ${ }^{1}$ \\ ${ }^{1}$ Department of Surgery, The University of Hong Kong, Hong Kong, SAR; ${ }^{2}$ Department of Otolaryngology, \\ The First People's Hospital of Foshan, Foshan, Guangdong 528000, P.R. China
}

Received June 7, 2016; Accepted October 26, 2016

DOI: $10.3892 / o r .2017 .5354$

\begin{abstract}
Radiation therapy is the standard treatment for primary nasopharyngeal carcinoma (NPC). MicroRNA regulates cancer responsiveness to radiation therapy by controlling the genes involved in radiation responses. Recent studies suggested that downregulation of microRNA-138-5p was clinically significant in NPC. Here, we evaluated the effect of miR-138-5p on radiosensitivity of NPC cells and explored the underlying mechanisms by identifying its target gene that impacted sensitivity to radiation. Our results revealed that overexpression of miR-138-5p reduced the ability to form colonies, inhibited proliferation, and enhanced radiation-induced DNA damage and autophagy in NPC cells upon radiation treatment. By integrating predicted targets with the transcripts downregulated by miR-138-5p, EIF4EBP1 was identified to be a target gene of miR-138-5p. Results from luciferase reporter assay demonstrated that miR-138-5p downregulated the expression of EIF4EBP1 by binding to the 3'-UTR. Silence of EIF4EBP1 enhanced radiosensitivity of NPC cells as evidenced by reduced ability to form colonies after radiation exposure. In summary, our results indicated that miR-138-5p enhanced radiosensitivity of NPC cells by targeting EIF4EBP1. Further studies are warranted to investigate the potential use of miR$138-5 \mathrm{p}$ in the clinical management and treatment prediction of NPC patients.
\end{abstract}

\section{Introduction}

Nasopharyngeal carcinoma (NPC) is a common head and neck cancer in southern China and Southeast Asia (1). At present, radiotherapy is the major treatment for patients with

Correspondence to: Dr Thian-Sze Wong, Department of Surgery, Queen Mary Hospital, 102 Pokfulam Road, Hong Kong SAR, P.R. China

E-mail: thiansze@gmail.com

${ }^{*}$ Contributed equally

Key words: nasopharyngeal carcinoma, microRNA-138-5p, radiation sensitivity, EIF4EBP1, DNA double-strand breaks undifferentiated NPC. Thus, the responsiveness of NPC cells to radiotherapy will have a direct impact on the treatment outcomes (2). The reasons underlying the presentation of radiation resistant phenotype in a subgroup of patients remain poorly understood. In case of radiation treatment failure, patients will have high chance to develop distant metastasis and recurrent disease (3-5). Although the use of molecular markers in predicting treatment outcome shows promising results in multiple solid tumors, the use of it in monitoring radiation response or prognostication remains limited in NPC (6). Thus, there is a need to identify key molecular markers with predictive value in the NPC patients.

MicroRNA is a group of non-coding RNA which functions as post-transcriptional regulator in controlling specific gene expression (7). Mature microRNA could form a thermodynamically stable duplex with the target mRNA by binding to the 3' untranslated region (UTR) or open reading frame (8). Binding to microRNA was able to hinder the translation process and suppress the corresponding protein level. In addition, the microRNA/mRNA duplex recruited Argonaute 2 (Ago2) forming the RNA-induced silencing complex (RISC) which subsequently promotes mRNA cleavage and degradation (8). MicroRNA dysregulation has been reported in nearly all the human malignancies. Cancers had differential microRNA expression profiles depending on the cellular context. The differentially expressed or suppressed microRNA could contribute to the pathological development of specific cancer phenotype by regulating characteristic gene expression.

MicroRNA-138-5p expression was significant reduced in NPC tissues and NPC cell lines (9). However, little is know about the functions of miR-138-5p in NPC. Liu et al performed a functional study on the role of miR-138-5p in NPC cell lines. They showed that re-introduction of miR-138-5p into the NPC cells could suppress the tumorigenicity of NPC cells in vivo (9). In this study, we addressed the functional role of miR-138-5p in the responsiveness of NPC cells to radiation treatment. Computational analysis showed that miR-138-5p could potentially target the transcript of eukaryotic initiation factor $4 \mathrm{E}$ binding protein 1 (EIF4EBP1). EIF4EBP1 is a translational regulator (10). EIF4EBP1 represses eukaryotic translation initiation factor 4E (eIF4E), the rate limiting cap-binding protein which regulates cap-dependent mRNA translation by binding to the 7-methyl-GTP 5' capped end of the mRNA (11). 
A recent study showed that increased EIF4EBP1 stability could promote therapeutic resistance in prostate cancer cell (12). Therefore, we suggest that miR-138-5p could modulate the responsiveness to radiation treatment by targeting EIF4EBP1 in NPC.

\section{Materials and methods}

Cell culture. Human NPC cell lines namely HONE1 and HK1, were derived from poorly and well differentiated NPC, respectively $(13,14)$. HONE1 and HK1 cells were maintained in RPMI-1640 medium additionally supplemented with $10 \%$ fetal bovine serum, $200 \mathrm{U} / \mathrm{ml}$ penicillin $\mathrm{G}$ sodium, $200 \mu \mathrm{g} / \mathrm{ml}$ streptomycin sulfate, and $0.5 \mu \mathrm{g} / \mathrm{ml}$ amphotericin B in a $37^{\circ} \mathrm{C}$ humidified incubator with $5 \% \mathrm{CO}_{2}$. Cell irradiation was performed by Gammacell ${ }^{\circledR} 3000$ Elan system (Best Theratronics, Ottawa, ON, Canada).

miR-138 precursor transfection. HONE1 and HK1 cells were transiently transfected with miR-138 precursor and negative control (Applied Biosystems, Carlsbad, CA, USA) by HiPerFect reagent (Qiagen, Valencia, CA, USA) according to the manufacturer's protocol. After $72 \mathrm{~h}$ of transfection, cells were collected and the efficiency of miR-138 precursor transfection was determined.

Real-time quantitative RT-PCR (QPCR). Total RNA was extracted by TRIzol (Life Technologies, Grand Island, NY, USA) according to the protocol of the manufacturer. HighCapacity cDNA Reverse Transcription kit (Applied Biosystems) was used for reverse transcription. Transcript levels of miR-138-5p and U6 control snRNA were measured by TaqMan Gene Expression assays (Applied Biosystems). The PCR primer-probe pairs for EIF4EBP1 were as follows: forward, 5'-AGCCCTTCCAGTGATGAGC-3'; reverse, 5'-TGT CCATCTCAAACTGTGACTCTT-3'; probe no. 21 of Universal ProbeLibrary (Roche Applied Science, Indianapolis, IN, USA). Primers were synthesized by Integrated DNA Technologies (Coralville, IA, USA). Glyceraldehyde-3-phosphate dehydrogenase (GAPDH) was used as an endogenous control and quantified by Universal ProbeLibrary Human GAPD Gene assay (Roche Applied Science). The fold changes were calculated using $2^{-\Delta \Delta \mathrm{Ct}}$ method.

Colony formation assay. Colony formation assay was carried out in 6-well cell culture plate with 600 cells per well. Cells were irradiated at a single dose of 2,4 , or 6 Gy and allowed to grow for two weeks. Then, colonies were stained with $0.5 \%$ crystal violet after fixation with $70 \%$ ethanol and colonies with $>50$ cells were counted.

Proliferation assay. Proliferation assay was performed by using RTCA DP instrument of xCELLigence Real-Time Cell Analyzer (Roche Applied Science). Cells were inoculated into E-Plate 16 and were irradiated by Gammacell 3000 Elan system (Best Theratronics). The proliferation rates of HONE1 and HK1 cells were examined constantly.

$\gamma H 2 A X$ phosphorylation detection. HONE1 and HK1 cells were plated on chamber slides and irradiated at a single dose of 2, 4, or 6 Gy. Then, cells were washed, fixed and incubated with rabbit polyclonal anti- $\gamma \mathrm{H} 2 \mathrm{AX}$ antibodies (Abcam, Cambridge, UK) and $\mathrm{CF}^{\mathrm{TM}} 488 \mathrm{~A}$ Secondary Antibody Conjugates (Biotium, Hayward, CA, USA). Nucleus and F-actin were counterstained with DAPI (Life Technologies) and Alexa Fluor ${ }^{\circledR} 635$ phalloidin (Life Technologies) respectively. Images were taken under a fluorescence microscope (Nikon, Tokyo, Japan) and the number of $\gamma \mathrm{H} 2 \mathrm{AX}$ foci in the cells was counted.

Acridine orange (AO) staining. AO (Sigma-Aldrich, St. Louis, MO, USA) staining was performed to detect the formation of acidic vesicular organelles (AVOs) during autophagy process. Cells were stained with $\mathrm{AO}$ at the concentration of $4 \mu \mathrm{g} / \mathrm{ml}$ for $15 \mathrm{~min}$ at $37^{\circ} \mathrm{C}$. Stained cells were observed by a fluorescent microscope (Nikon).

Microarray. Affymetrix HG-U133 Plus 2 array (Affymetrix, Santa Clara, CA, USA) was used to detect global gene expression profiling. The quality of total RNA was evaluated using Agilent 2100 Bioanalyzer (Agilent Technologies). Data analysis was performed using GeneSpring version 13 (Agilent Technologies). Microarray was conducted by the Centre for Genomic Sciences, the University of Hong Kong.

Luciferase reporter assay. The sense and antisense strands of wild-type and mutant 3'-UTR of EIF4EBP1 were synthesized. The oligonucleotides were as follows: wild-type sense strand: 5'-CCAGGGCACCTGCCCCCTCCTCTTCGTGAACACCA GCAGATACCTCCTTGTGA-3'; wild-type antisense strand: 5'-AGCTTCACAAGGAGGTATCTGCTGGTGTTCACGAA GAGGAGGGGGCAGGTGCCCTGGAGCT-3'; mutant sense strand: 5'-CCAGGGCACCTGCCCCCTCCTCTTCGTGA AAGTACTAAGATACCTCCTTGTGA-3'; mutant antisense strand: 5'-AGCTTCACAAGGAGGTATCTTAGTACTTTC ACGAAGAGGAGGGGGCAGGTGCCCTGGAGCT-3'. The annealed sense and antisense strands of wild-type or mutant 3'-UTR of EIF4EBP1 were cloned into the SacI and HindIII sites of pMIR-Report Luciferase vector (Applied Biosystems) to produce Luc-wild-type or Luc-mutant construct, respectively. HONE1 cells were co-transfected with $200 \mathrm{ng}$ Luc-wild-type vector or Luc-mutant vector, $50 \mathrm{nM}$ miR-138 precursor or negative control (Applied Biosystems), together with $200 \mathrm{ng}$ pMIR-Report $\beta$-galactosidase control vector (Applied Biosystems) using Lipofectamine 2000 (Life Technologies). After $48 \mathrm{~h}$, firefly luciferase and $\beta$-galactosidase activities were determined using Dual-Light luminescent reporter gene assay kit (Applied Biosystems) on an LB 96V microplate luminometer (EG\&G Berthold, Bad Wildbad, Germany).

siRNA transfection. EIF4EBP1 siRNA-1, EIF4EBP1 siRNA-2 and negative control siRNA (Qiagen) were transfected into HONE1 and HK1 cells using HiPerFect transfection reagent (Qiagen) in accordance with the protocol from the manufacturer. After $72 \mathrm{~h}$, cells were collected and the efficiency of EIF4EBP1 silencing was determined by QPCR.

Statistical analysis. Student's t-test was employed to compare quantitative variables between two groups. All the tests were 


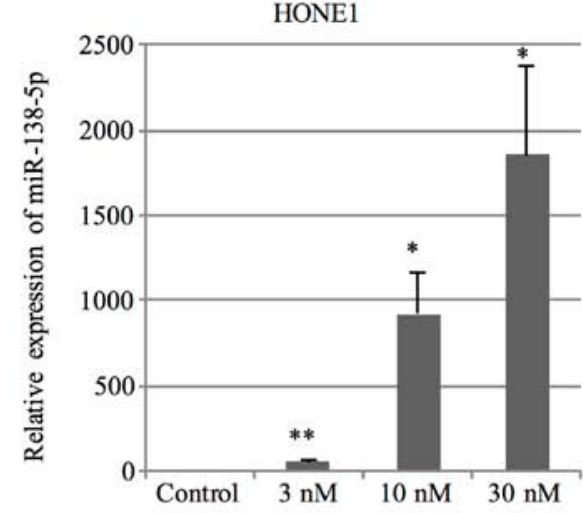

HK1

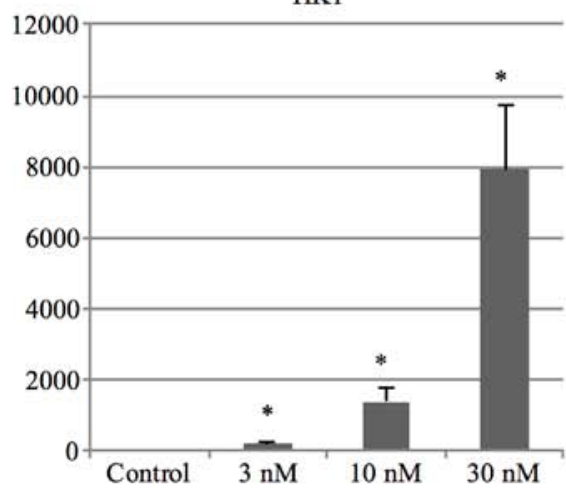

Concentration of miR-138 precursor

B

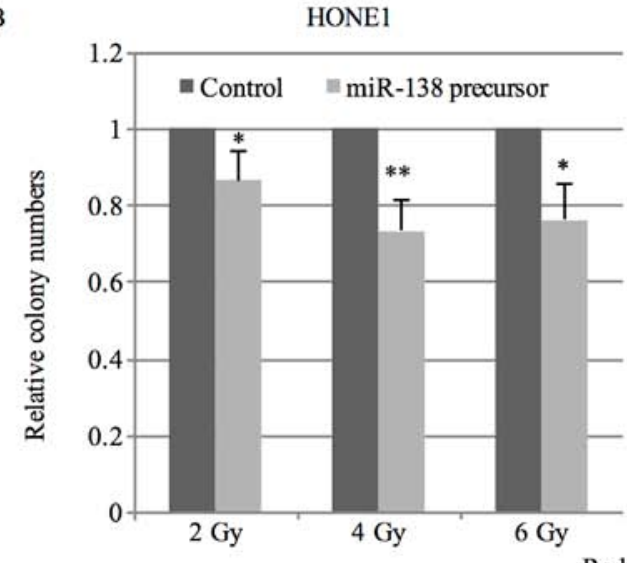

HK1

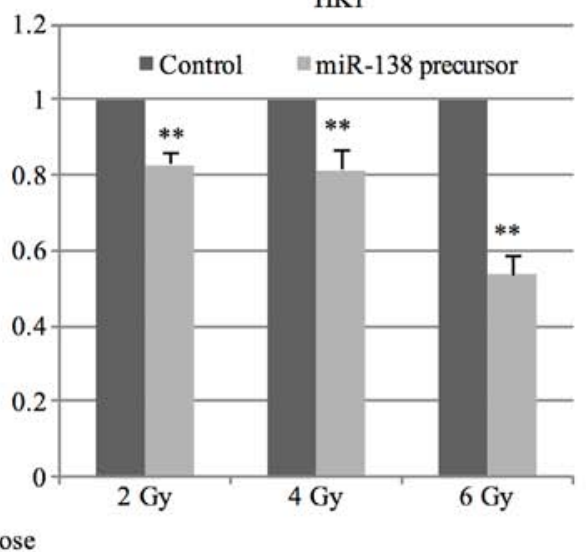

C

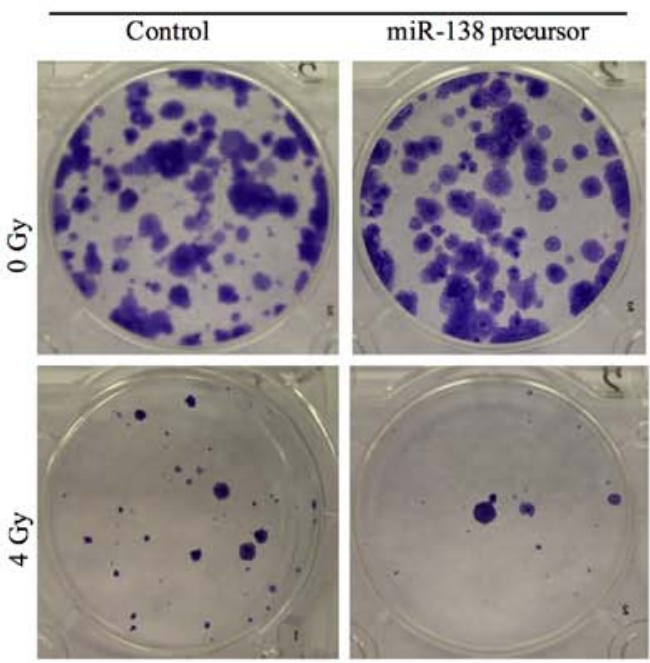

HK1

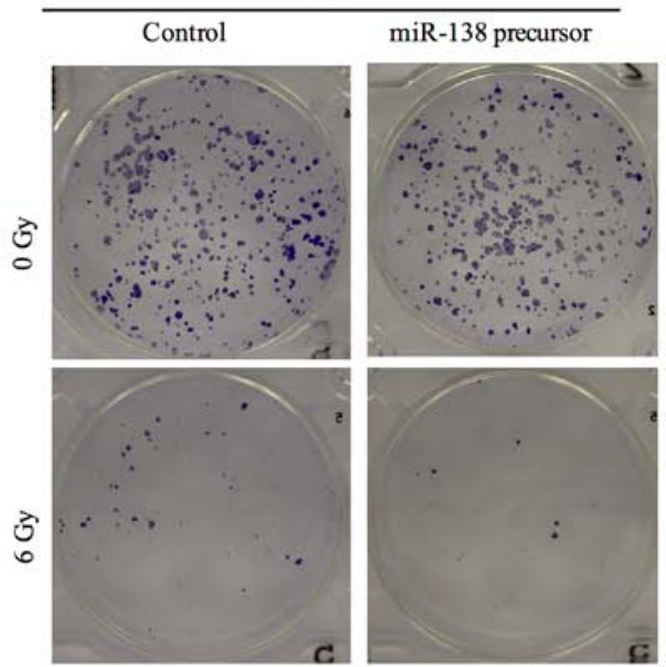

Figure 1. Overexpression of miR-138-5p enhanced the sensitivity of NPC cells to radiation. (A) QPCR analysis of miR-138-5p expression level in NPC cells transfected with miR-138 precursor. (B) Colony formation assay of NPC cells transfected with miR-138 precursor upon different doses of radiation treatment. (C) Representative images showing colony formation. Error bar indicate $\mathrm{SD}, \mathrm{n}=3,{ }^{*} \mathrm{p}<0.05,{ }^{* *} \mathrm{p}<0.01$.

2-sided. Results were only considered statistically significant if $\mathrm{p}<0.05$.

\section{Results}

Overexpression of miR-138-5p enhanced the sensitivity of $N P C$ cells to radiation. To evaluate the effect of miR-138-5p on radiosensitivity of NPC cells, we overexpressed miR-138-5p by transfection of miR-138 precursor. A dose-dependent increase in miR-138-5p expression was observed (Fig. 1A). Colony formation assay was performed to assess the sensitivity to radiation treatment. Cells transfected with $10 \mathrm{nM}$ miR-138 precursor or negative control were exposed to radiation at the dose of 2,4 or $6 \mathrm{~Gy}$ and the survival fraction was counted after 

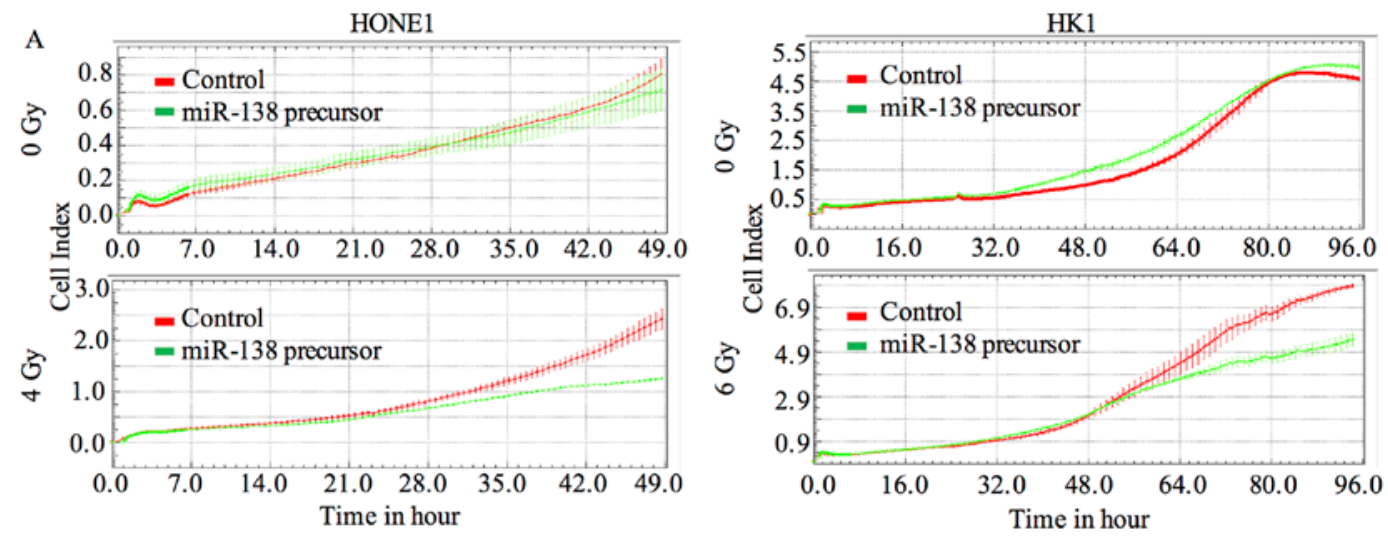

B

HONE1

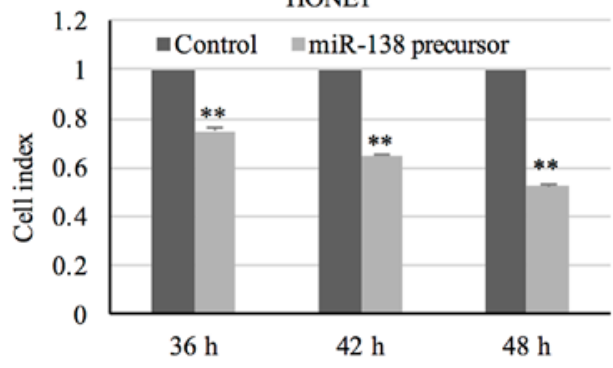

HK1

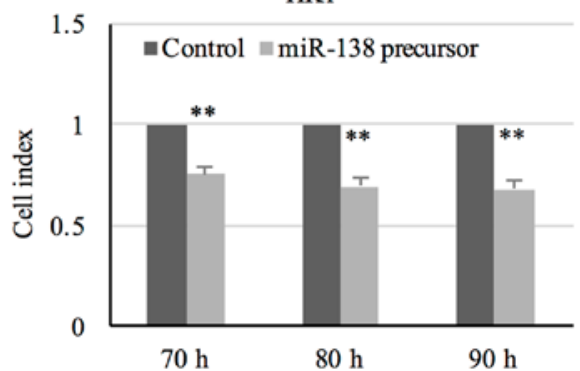

$\mathrm{C}$
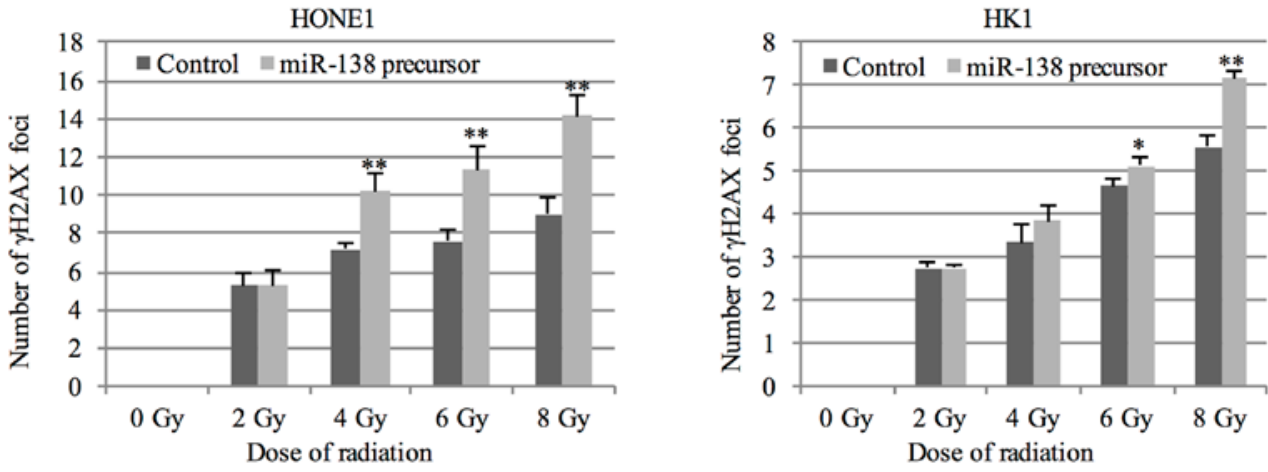

D
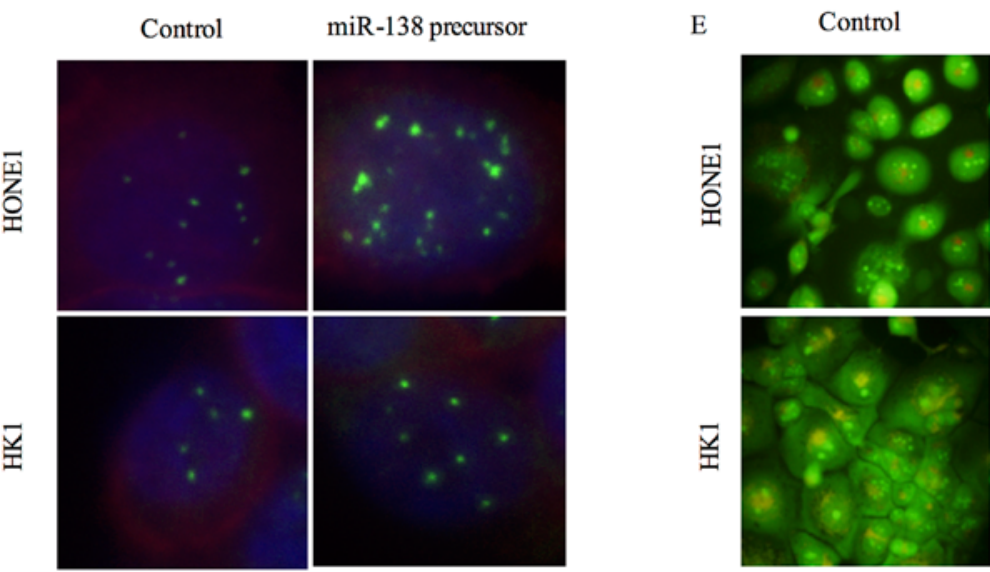

miR-138 precursor

Figure 2. Overexpression of miR-138-5p inhibited proliferation and enhanced the $\gamma \mathrm{H} 2 \mathrm{AX}$ foci formation and autophagy of NPC cells under radiation treatment. (A) Real-time monitoring of proliferation rate of NPC cells transfected with miR-138 precursor exposed to radiation treatment using xCELLigence Real-Time Cell Analyzer. Error bar indicates SD, n=3. (B) Cell index at different time-points. Error bar indicates SD, n=3. (C) The number of $\gamma \mathrm{H} 2 \mathrm{AX}$ foci of NPC transfected with miR-138 precursor upon radiation treatment. Error bar indicates SE, $n=20$. (D) Representative images showing $\gamma \mathrm{H} 2 \mathrm{AX}$ foci formation. (E) Representative images showing formation of acidic vesicular organelles in NPC cells transfected with miR-138 precursor exposed to radiation treatment. ${ }^{*} \mathrm{p}<0.05,{ }^{* *} \mathrm{p}<0.01$.

14 days. An apparent decrease in colony number was observed in cells transfected with miR-138 precursor in comparison with negative control under radiation treatment (Fig. 1B and C).
Overexpression of $m i R-138-5 p$ inhibited proliferation and enhanced the $\gamma H 2 A X$ foci formation and autophagy of NPC cells under radiation treatment. The proliferation rate after 


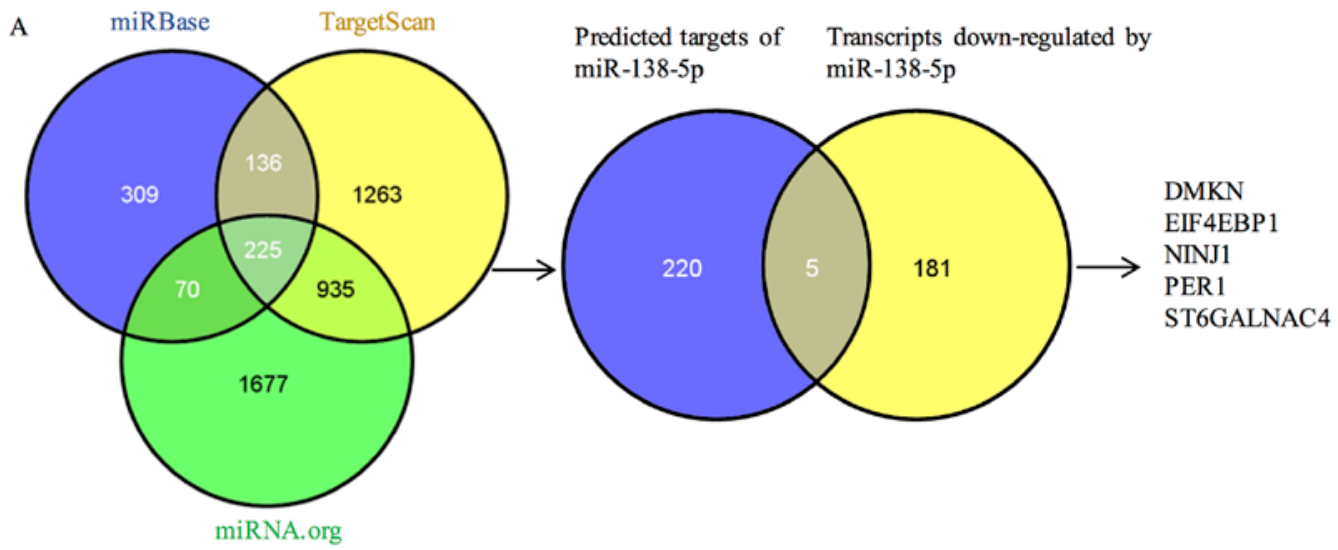

B
3'- G C C G G A C U A A G U G - U U G U G G U C G A -5' miR-138-5p
\begin{tabular}{lll|l|l|l|l|l|}
$|l|$ & $\mid$
\end{tabular}
5'- C C U C C U - C U U C G U G A A C A C C A G C A -3' Wild-type EIF4EBP1 (158 bp-180 bp)
5'- C C U C C U - C U U C G U G A A A G T A C T A A -3' Mutant EIF4EBP1 (158 bp-180 bp)

C

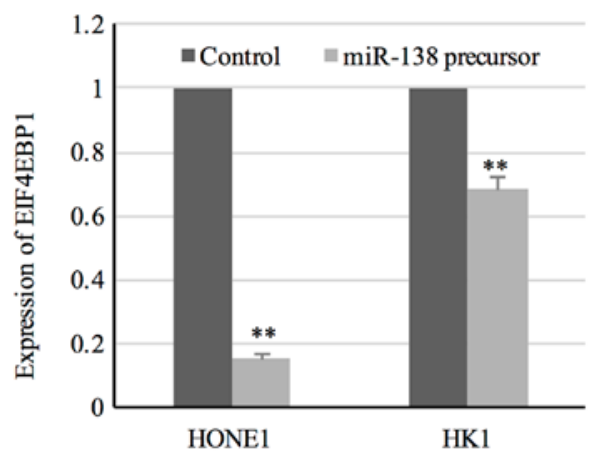

E

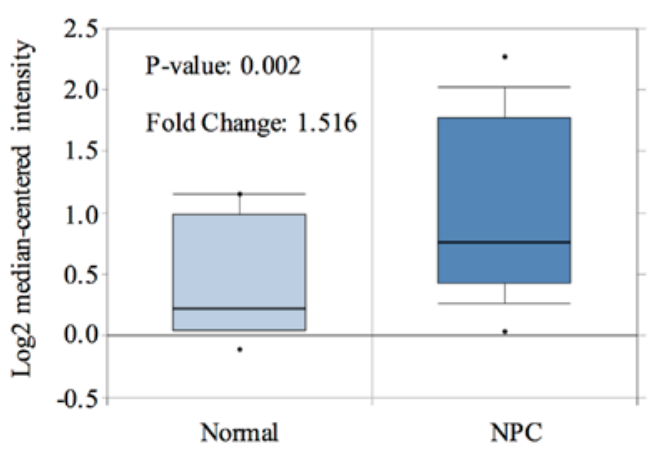

D

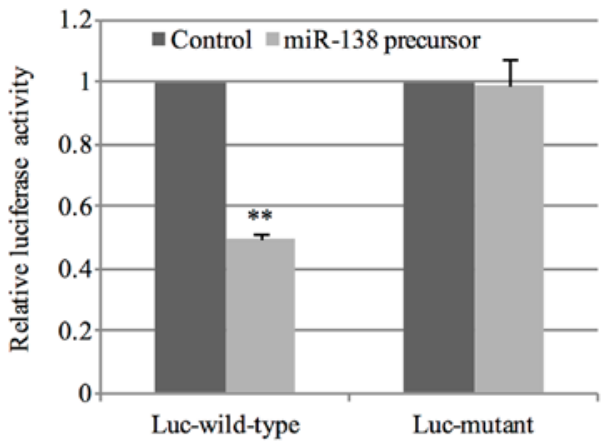

Figure 3. miR138-5p directly targets EIF4EBP1. (A) Integration of predicted targets of miR-138-5p and transcripts downregulated by miR-138-5p from microarray analysis identified EIF4EBP1 as a putative direct target of miR-138-5p. (B) The predicted miR-138-5p binding sequence located in the 3'-UTR of EIF4EBP1 and the mutation of the miR-138-5p binding sequence for luciferase reporter assay. (C) QPCR detection of EIF4EBP1 expression in NPC cells transfected with miR-138 precursor. (D) Luciferase reporter assay of HONE1 cells co-transfected with Luc-wild-type vector or Luc-mutant vector and miR-138 precursor or negative control. Error bar indicates SD, $n=3,{ }^{* *} \mathrm{p}<0.01$. (E) Expression level of EIF4EBP1 in NPC and normal tissues in GSE12452 from Oncomine.

radiation treatment was used to assess the radiosensitivity. Cells transfected with $10 \mathrm{nM}$ miR-138 precursor were exposed to radiation at 24 hours after transfection and cell proliferation was continuously monitored. Although miR-138 precursor itself did not affect the proliferation rate of cells without radiation exposure, cells transfected with miR-138 precursor exhibited a significant reduction in proliferation rate in a time-dependent manner under radiation treatment (Fig. 2A and B). Radiation killed cells by inducing DNA double-strand breaks (DSBs). DSBs were recognized by varied protein complexes including $\gamma \mathrm{H} 2 \mathrm{AX}$. Staining of cells with anti- $\gamma \mathrm{H} 2 \mathrm{AX}$ antibody generated foci corresponding to DSBs in nuclei (15). Overexpression of miR-138-5p resulted in an obvious increase in the number of $\gamma \mathrm{H} 2 \mathrm{AX}$ foci after radiation treatment (Fig. 2C and D), indicating a higher degree of radiation-induced DNA damage. Radiation could induce autophagy in cancer cells. Formation of AVOs was a characteristic feature of autophagy. In AO-stained cells, cytoplasm and nucleolus displayed green fluorescence. In contrast, AVOs fluoresced bright red (16). An increase in red signal was observed in NPC cells transfected with miR-138 precursor compared to negative 
control after exposure to radiation (Fig. 2E), implicating a higher level of radiation-induced autophagy.

miR138-5p directly targets EIF4EBP1. First, we used prediction databases to predict the targets of miR-138-5p. Considering that individual prediction database probably generated false positive targets, we employed Bioinformatics resource manager v2.3 (BRM) software (http://www.sysbio. org/dataresources/brm.stm) for prediction (17). BRM utilizes three databases including microCosm/miRBase, TargetScan and miRNA.org to predict targets. Those targets that are conserved across all three databases were considered as high-confidence ones. By the prediction function of BRM, 225 genes were predicted to be targets of miR-138-5p by all three databases. Given that miRNA promoted the degradation of target mRNA, we carried out microarray analysis to identify transcripts downregulated by miR-138-5p, which were its putative targets. The global gene expression profiling of HONE1 cells transfected with miR-138 precursor was examined. With a fold-change cut-off of 3 and $p<0.05,186$ genes were found to be downregulated by miR-138-5p. Then, the 225 high-confidence targets were integrated with the 186 transcripts downregulated by miR-138-5p. By this novel strategy, we identified 5 potential targets including DMKN, EIF4EBP1, NINJ1, PER1 and ST6GALNAC4 (Fig. 3A).

The binding sites between miR-138-5p and 3'-UTR of EIF4EBP1 are shown in Fig. 3B. Results from QPCR further confirmed the downregulation of EIF4EBP1 by miR-138-5p (Fig. 3C). To explore whether miR-138-5p modulated the expression of EIF4EBP1 by binding to the 3'-UTR, luciferase reporter assay was performed in HONE1 cells transfected with luciferase vector containing 3'-UTR of EIF4EBP1 together with miR-138 precursor or negative control. Overexpression of miR-138-5p significantly reduced the luciferase activity of luciferase vector harboring wild-type 3'-UTR of EIF4EBP1 (Fig. 3D). In contrast, mutation of the binding sites within 3'-UTR of EIF4EBP1 abrogated the suppressing effect (Fig. 3D).

To gain insights into the clinical implications of EIF4EBP1 in NPC, the expression of EIF4EBP1 was analyzed in publicly available microarray dataset GSE12452 in the Oncomine database (www.oncomine.org) (18). GSE12452 contained expression data of 31 NPC tissues and 10 normal controls. A 1.516-fold increase in EIF4EBP1 expression was observed in NPC tissues in comparison with normal controls (Fig. 3E).

Silence of EIF4EBPI increased the sensitivity of NPC cells to radiation. To investigate the role of EIF4EBP1 in radiosensitivity of NPC cells, the expression of EIF4EBP1 was silenced by 2 specific siRNA. Transfection of siRNA at the concentration of $2.5 \mathrm{nM}$ successfully inhibited the expression of EIF4EBP1 in HONE1 cells, while siRNA at the concentration of $10 \mathrm{nM}$ was required to suppress the expression in HK1 cells (Fig. 4A). NPC cells transfected with EIF4EBP1 siRNA were exposed to radiation treatment at the dose of 2,4 or $6 \mathrm{~Gy}$ and the ability to form colony was measured after 14 days. NPC cells with silenced expression of EIF4EBP1 displayed a significant decrease in the number of colonies after radiation treatment compared to negative control siRNA (Fig. 4B and C). Results from AO staining showed that silence of EIF4EBP1 enhanced radiation-induced autophagy as evidenced by an increase in red signal under radiation treatment (Fig. 4D).

\section{Discussion}

We found that expression of miR-138-5p is one of the determining factors involved in modulating the sensitivity of NPC cells to radiation treatment. miR-138-5p controls the cell cycle related genes in hepatocellular carcinoma (19). In NPC, the oncogenic cell cycle regulator CCND1 is a reported target of miR-138-5p (9). In small cell lung cancer, inhibition of cell cycle progression is also observed in miR-138-5p overexpressing cells (20). It is suggested that miR-138-5p might be involved in DNA damage response by targeting the histone H2AX (20). Recently, the role of miR-138-5p in modulating the sensitivity to cancer therapeutics is reported. In prostate cancer, miR-138-5p regulates Kindlin-2, a regulator of integrin adhesion receptor which is frequently overexpressed in castration-resistant prostate cancer (21). Suppressing Kindlin-2 could improve the sensitivity of prostate cancer cells to docetaxel (21). Upregulating miR-138-5p could increase the responsiveness of lung cancer cells to radiation treatment (22).

eIF4E is an oncogenic driver which could promote malignant transformation $(23,24)$. Increased eIF4F expression is rarely found in the benign lesions (25). High expression has been observed in cancer with aggressive cancer and poorly differentiated phenotype (26). Overexpression of eIF4F is also found in cancers of the head and neck regions including oral cavity, oropharynx, larynx and nasopharynx (27-29). In NPC, high eIF4F expression promotes proliferation and cell cycle progression with high correlation to the oncogenic protein c-Myc and MMP9 (29). As the cancer cell responses to radiation damage by activating the cell cycle checkpoints, it is suggested that eIF4E might function in radiation response by initiating expression of genes involved in cell cycle regulation $(30,31)$. Apart from the protein translation function, eIF4E can promote oncogenic transformation by preferential export of capped mRNAs containing 50-nucleotide structural element in the $3^{\prime}$ UTR (32).

Inactivation of the eIF4E inhibitor EIF4EBP1 could be achieved by phosphorylation. The serine/threonine protein kinase mammalian target of rapamycin (mTOR) is a critical player involved in EIF4EBP1 phosphorylation and dissociating from eIF4E (33). Another serine/threonine protein kinase $\mathrm{Akt}$, upstream of mTOR, is an indicator of poor prognosis of NPC patients. Activated Akt showed high correlation with the inactivated EIF4EBP1, which is a strong indication of the involvement of Akt signaling in EIF4EBP1 regulation (34). Akt/mTOR signaling can be activated by radiation. Thus, the use of Akt/mTOR inhibitors could increase the sensitivity of cancer cells to radiation (35).

In general, hypoxic cells are less sensitive to radiation treatment due to the specific expression of particular proteins in the hypoxic environment (36). It has been shown that EIF4EBP1 expression will affect the cancer proteomic leading to the differential expression of different proteins under normoxic and hypoxic conditions (37). The quantity of EIF4EBP1 protein and activated EIF4EBP1 was induced in response to radiation treatment (38). In glioblastoma, inhibiting EIF4EBP1 could sensitize the xenograft to radiation (39). Thus, it is important 
A

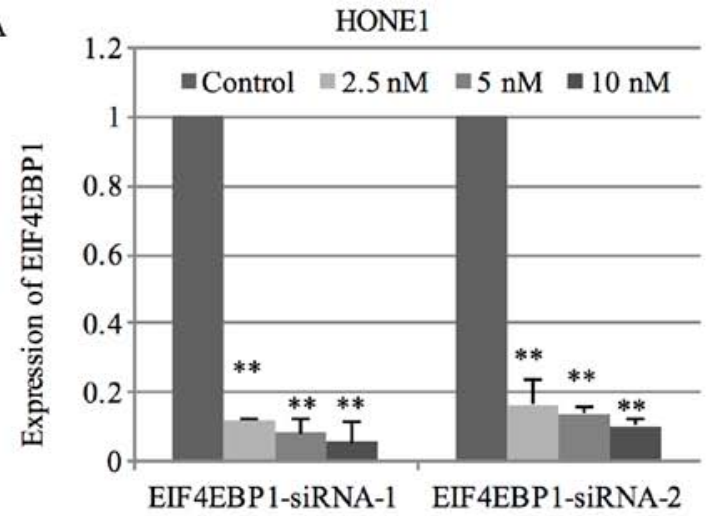

B

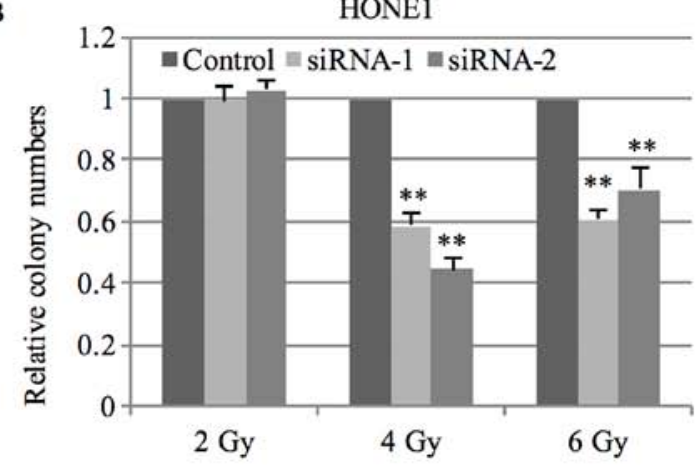

$\mathrm{C}$

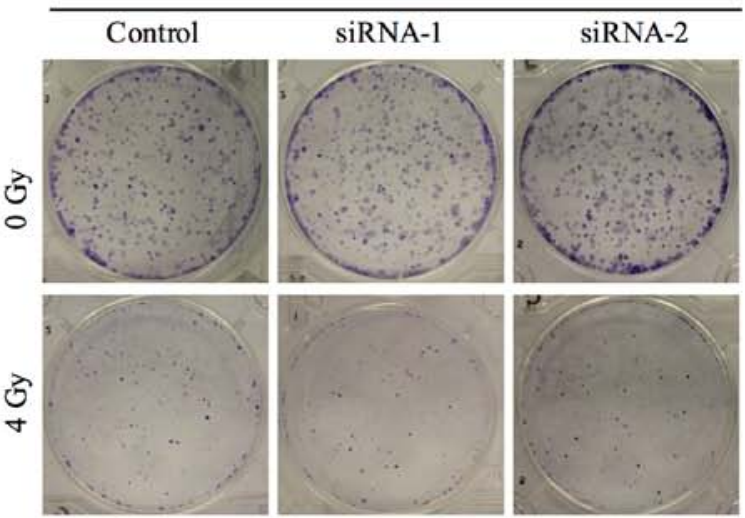

D

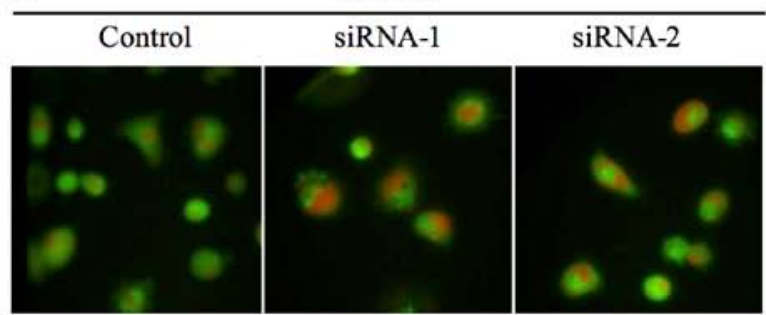

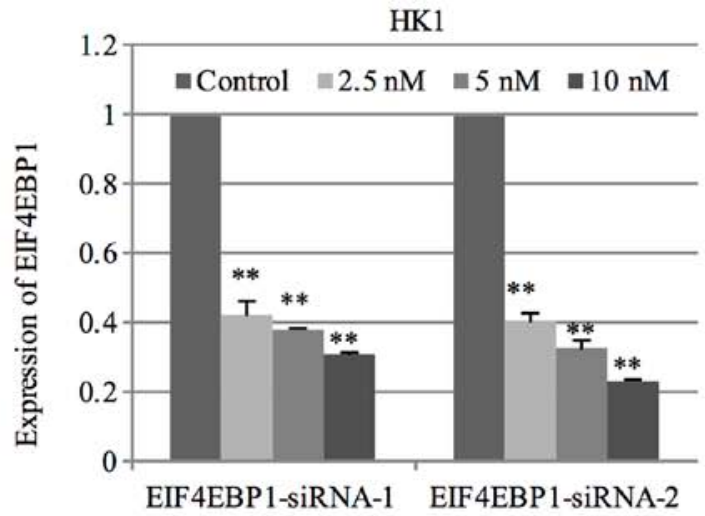
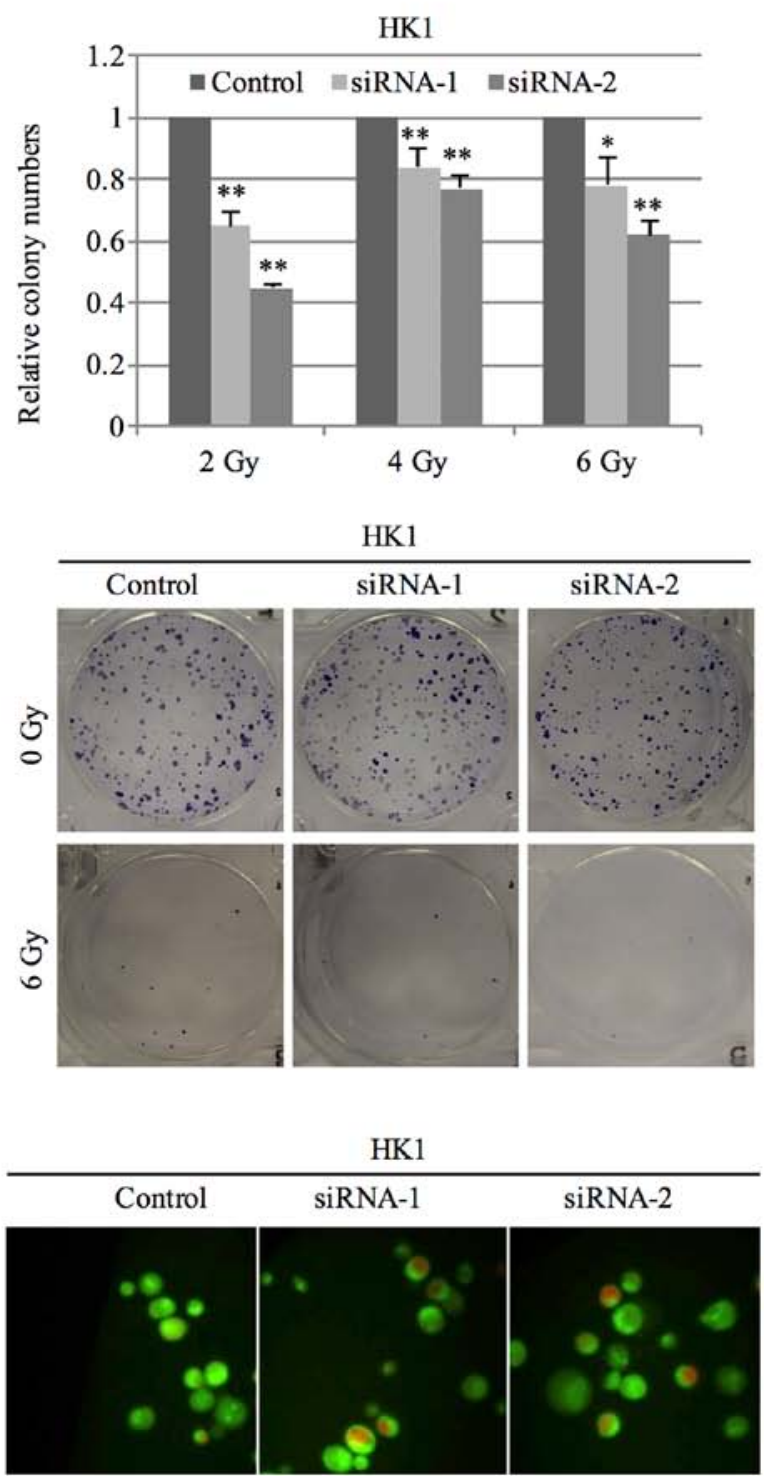

Figure 4. Silence of EIF4EBP1 increased the sensitivity of NPC cells to radiation. (A) QPCR analysis of EIF4EBP1 expression level in NPC cells transfected with EIF4EBP1 siRNA. (B) Colony formation assay of NPC cells transfected with EIF4EBP1 siRNA upon different doses of radiation treatment. (C) Representative images showing colony formation. (D) Representative images showing formation of acidic vesicular organelles in NPC cells transfected with EIF4EBP1 siRNA upon radiation treatment. Error bar indicates $\mathrm{SD}, \mathrm{n}=3,{ }^{*} \mathrm{p}<0.05,{ }^{* *} \mathrm{p}<0.01$.

to explore the functional impact and the role of EIF4EBP1 in the radiation responses.

In conclusion, our results indicate that miR-138-5p mediates the responsiveness of NPC to ionizing radiation by targeting
EIF4EBP1. Further study on the impact of miR-138-5p and EIF4EBP1 on the radiosensitivity of tumor cells will be useful for the development of therapeutic regimens to enhance the therapeutic radiosensitivity of NPC. 


\section{Acknowledgements}

This study was supported by S.K. Yee Medical Foundation Grant and Seed Funding of Basic Research, The University of Hong Kong.

\section{References}

1. Wei WI and Sham JS: Nasopharyngeal carcinoma. Lancet 365: 2041-2054, 2005.

2. Feng XP, Yi H, Li MY, Li XH, Yi B, Zhang PF, Li C, Peng F, Tang CE, Li JL, et al: Identification of biomarkers for predicting nasopharyngeal carcinoma response to radiotherapy by proteomics. Cancer Res 70: 3450-3462, 2010.

3. Leung SF, Teo PM, Shiu WW, Tsao SY and Leung TW: Clinical features and management of distant metastases of nasopharyngeal carcinoma. J Otolaryngol 20: 27-29, 1991.

4. Lee AW, Poon YF, Foo W, Law SC, Cheung FK, Chan DK, Tung SY, Thaw M and Ho JH: Retrospective analysis of 5037 patients with nasopharyngeal carcinoma treated during 1976-1985: Overall survival and patterns of failure. Int J Radiat Oncol Biol Phys 23: 261-270, 1992.

5. Chan JY, Chow VL, Tsang R and Wei WI: Nasopharyngectomy for locally advanced recurrent nasopharyngeal carcinoma: Exploring the limits. Head Neck 34: 923-928, 2012.

6. Lee AW, Ng WT, Chan YH, Sze H, Chan C and Lam TH: The battle against nasopharyngeal cancer. Radiother Oncol 104: 272-278, 2012.

7. Garzon R, Calin GA and Croce CM: MicroRNAs in cancer. Annu Rev Med 60: 167-179, 2009.

8. Shenouda SK and Alahari SK: MicroRNA function in cancer: Oncogene or a tumor suppressor? Cancer Metastasis Rev 28: 369-378, 2009.

9. Liu X, Lv XB, Wang XP, Sang Y, Xu S, Hu K, Wu M, Liang Y, Liu P, Tang J, et al: MiR-138 suppressed nasopharyngeal carcinoma growth and tumorigenesis by targeting the CCND1 oncogene. Cell Cycle 11: 2495-2506, 2012.

10. Pause A, Belsham GJ, Gingras AC, Donzé O, Lin TA, Lawrence JC Jr and Sonenberg N: Insulin-dependent stimulation of protein synthesis by phosphorylation of a regulator of 5'-cap function. Nature 371: 762-767, 1994.

11. Meric F and Hunt KK: Translation initiation in cancer: A novel target for therapy. Mol Cancer Ther 1: 971-979, 2002.

12. Yu L, Shang ZF, Wang J, Wang H, Huang F, Zhang Z, Wang Y, Zhou J and Li S: PC-1/PrLZ confers resistance to rapamycin in prostate cancer cells through increased 4E-BP1 stability. Oncotarget 6: 20356-20369, 2015.

13. Glaser R, Zhang HY, Yao KT, Zhu HC, Wang FX, Li GY, Wen DS and Li YP: Two epithelial tumor cell lines (HNE-1 and HONE-1) latently infected with Epstein-Barr virus that were derived from nasopharyngeal carcinomas. Proc Natl Acad Sci USA 86: 9524-9528, 1989.

14. Huang DP, Ho JH, Poon YF, Chew EC, Saw D, Lui M, Li CL, Mak LS, Lai SH and Lau WH: Establishment of a cell line (NPC/HK1) from a differentiated squamous carcinoma of the nasopharynx. Int J Cancer 26: 127-132, 1980.

15. Kuo LJ and Yang LX: Gamma-H2AX - a novel biomarker for DNA double-strand breaks. In Vivo 22: 305-309, 2008.

16. Paglin S, Hollister T, Delohery T, Hackett N, McMahill M, Sphicas E, Domingo D and Yahalom J: A novel response of cancer cells to radiation involves autophagy and formation of acidic vesicles. Cancer Res 61: 439-444, 2001.

17. Tilton SC, Tal TL, Scroggins SM, Franzosa JA, Peterson ES, Tanguay RL and Waters KM: Bioinformatics Resource Manager v2.3: An integrated software environment for systems biology with microRNA and cross-species analysis tools. BMC Bioinformatics 13: 311, 2012.

18. Rhodes DR, Yu J, Shanker K, Deshpande N, Varambally R, Ghosh D, Barrette T, Pandey A and Chinnaiyan AM: ONCOMINE: A cancer microarray database and integrated data-mining platform. Neoplasia 6: 1-6, 2004.

19. Wang W, Zhao LJ, Tan YX, Ren H and Qi ZT: MiR-138 induces cell cycle arrest by targeting cyclin D3 in hepatocellular carcinoma. Carcinogenesis 33: 1113-1120, 2012.
20. Yang H, Luo J, Liu Z, Zhou R and Luo H: MicroRNA-138 regulates DNA damage response in small cell lung cancer cells by directly targeting H2AX. Cancer Invest 33: 126-136, 2015.

21. Sossey-Alaoui K and Plow EF: miR-138-mediated regulation of KINDLIN-2 expression modulates sensitivity to chemotherapeutics. Mol Cancer Res 14: 228-238, 2016.

22. Yang H, Tang Y, Guo W, Du Y, Wang Y, Li P, Zang W, Yin X, Wang $\mathrm{H}$, Chu $\mathrm{H}$, et al: Up-regulation of microRNA-138 induce radiosensitization in lung cancer cells. Tumour Biol 35: 6557-6565, 2014.

23. Larsson O, Li S, Issaenko OA, Avdulov S, Peterson M, Smith K, Bitterman PB and Polunovsky VA: Eukaryotic translation initiation factor $4 \mathrm{E}$ induced progression of primary human mammary epithelial cells along the cancer pathway is associated with targeted translational deregulation of oncogenic drivers and inhibitors. Cancer Res 67: 6814-6824, 2007.

24. Avdulov S, Li S, Michalek V, Burrichter D, Peterson M, Perlman DM, Manivel JC, Sonenberg N, Yee D, Bitterman PB, et al: Activation of translation complex eIF4F is essential for the genesis and maintenance of the malignant phenotype in human mammary epithelial cells. Cancer Cell 5: 553-563, 2004.

25. Zheng J, Li J, Xu L, Xie G, Wen Q, Luo J, Li D, Huang D and Fan S: Phosphorylated Mnk1 and eIF4E are associated with lymph node metastasis and poor prognosis of nasopharyngeal carcinoma. PLoS One 9: e89220, 2014.

26. De Benedetti A and Harris AL: eIF4E expression in tumors: Its possible role in progression of malignancies. Int J Biochem Cell Biol 31: 59-72, 1999.

27. Nathan CO, Franklin S, Abreo FW, Nassar R, de Benedetti A, Williams J and Stucker FJ: Expression of eIF4E during head and neck tumorigenesis: Possible role in angiogenesis. Laryngoscope 109: 1253-1258, 1999.

28. Sorrells DL Jr, Ghali GE, De Benedetti A, Nathan CO and Li BD: Progressive amplification and overexpression of the eukaryotic initiation factor $4 \mathrm{E}$ gene in different zones of head and neck cancers. J Oral Maxillofac Surg 57: 294-299, 1999.

29. Wu M, Liu Y, Di X, Kang H, Zeng H, Zhao Y, Cai K, Pang T, Wang S, Yao Y, et al: EIF4E over-expresses and enhances cell proliferation and cell cycle progression in nasopharyngeal carcinoma. Med Oncol 30: 400, 2013.

30. Pawlik TM and Keyomarsi K: Role of cell cycle in mediating sensitivity to radiotherapy. Int J Radiat Oncol Biol Phys 59: 928-942, 2004.

31. Culjkovic B, Topisirovic I, Skrabanek L, Ruiz-Gutierrez M and Borden KL: eIF4E is a central node of an RNA regulon that governs cellular proliferation. J Cell Biol 175: 415-426, 2006.

32. Culjkovic-Kraljacic B, Baguet A, Volpon L, Amri A and Borden KL: The oncogene eIF4E reprograms the nuclear pore complex to promote mRNA export and oncogenic transformation. Cell Rep 2: 207-215, 2012.

33. Goberdhan DCI and Boyd CAR: mTOR: Dissecting regulation and mechanism of action to understand human disease. Biochem Soc Trans 37: 213-216, 2009.

34. Wang W, Wen Q, Xu L, Xie G, Li J, Luo J, Chu S, Shi L, Huang D, $\mathrm{Li} \mathrm{J}$, et al: Activation of Akt/mTOR pathway is associated with poor prognosis of nasopharyngeal carcinoma. PLoS One 9: e106098, 2014.

35. Albert JM, Kim KW, Cao C and Lu B: Targeting the Akt/ mammalian target of rapamycin pathway for radiosensitization of breast cancer. Mol Cancer Ther 5: 1183-1189, 2006.

36. Brown JM: The hypoxic cell: A target for selective cancer therapy - eighteenth Bruce F. Cain Memorial Award lecture. Cancer Res 59: 5863-5870, 1999.

37. Magagnin MG, van den Beucken $T$, Sergeant K, Lambin $P$, Koritzinsky M, Devreese B and Wouters BG: The mTOR target 4E-BP1 contributes to differential protein expression during normoxia and hypoxia through changes in mRNA translation efficiency. Proteomics 8: 1019-1028, 2008.

38. Braunstein S, Badura ML, Xi Q, Formenti SC and Schneider RJ: Regulation of protein synthesis by ionizing radiation. Mol Cell Biol 29: 5645-5656, 2009.

39. Dubois L, Magagnin MG, Cleven AH, Weppler SA, Grenacher B, Landuyt W, Lieuwes N, Lambin P, Gorr TA, Koritzinsky M, et al: Inhibition of 4E-BP1 sensitizes U87 glioblastoma xenograft tumors to irradiation by decreasing hypoxia tolerance. Int $\mathbf{J}$ Radiat Oncol Biol Phys 73: 1219-1227, 2009. 